\title{
Application of artificial intelligence in pediatric ophthalmic practice
}

\author{
Mirosława Grałek, Anna Niwald \\ Department of Pediatric Ophthalmology, M. Konopnicka Pediatrics Center, Independent Public Healthcare Center - Central Teaching Hospital of \\ the Medical University of Lodz, Poland
}

\begin{abstract}
Artificial intelligence (AI) has been developing rapidly in recent years, and finding its way into a number of areas of activity. In ophthalmology, AI has been used since digital information supplied by color fundus photography, optical coherence tomography, computerized visual field testing, and other techniques became available also in this field of medicine. Such information is based on an enormous pool of data forming a basis for machine learning and deep learning. Pediatric ophthalmology is a branch of ophthalmology which has also been increasingly benefiting from
\end{abstract}

the possibilities created by artificial intelligence. The goal of AI in ophthalmology is the early detection of diseases in which prompt treatment can prevent a permanent decrease in visual acuity. AIbased screening in children carries a particular value in preventing and monitoring retinopathy of prematurity, cataract, and myopia. In addition, artificial intelligence is useful in post-operative patient management.

KEY WORDS: artificial intelligence, child, ocular diseases, diagnostics, prophylaxis.

\section{ARTIFICIAL INTELLIGENCE}

Despite being known for over half a century, artificial intelligence (AI) is currently an ever-popular topic of academic research and multiple practical applications in many different areas of life, including multifunctional AI platforms used in medicine. The rapid development of AI during the last decade is linked to the development of advanced image processing methods and the implementation of new computer techniques. The term "artificial intelligence" was probably first used by John McCarthy, an American computer scientist who in 1955 referred to AI as "the science and engineering of making intelligent machines" [1]. In contemporary times, $\mathrm{AI}$ is defined as the ability of a system to correctly interpret external data, learn from the data it has been supplied with, and use these experiences to achieve specific goals and tasks by adapting flexibly to the implementation of the addressed problems [2]. With the aid of AI techniques, digital images providing sufficiently large numbers (thousands or even millions) of morphological datasets can be analyzed quickly and non-invasively in a comprehensive manner. The obtained results are determined not only by the number of examined data (datasets), but also by their representativeness of real-life images submitted for evaluation by artificial intelligence. Regardless of the degree of complexity and area of applica- tion, contemporary AI has two main components: machine learning (ML), also referred to as self-learning of machines or self-learning systems, and deep learning (DL). Machine learning is an interdisciplinary field of science that combines diverse areas such as computer science (e.g. programming, robotics, databases), and statistics (e.g. statistical data analysis).The main goal of ML is the practical application of AI achievements to create an automatic system that can improve itself through the accumulated experience (i.e. data) and, on this basis, acquire new knowledge and make decisions derived from appropriate algorithms. Deep learning is a method by which the computer "learns" by itself, relying on a database of selected data it has been supplied with, without direct human intervention. At present, artificial intelligence, using both ML and DL, also has applications in various medical disciplines. The widespread adoption of electronic health records has resulted in the accumulation of an extensive pool of clinical data that is being successfully used for deep learning.

Ophthalmology is keeping pace with other disciplines in the implementation of these automated techniques, with AI being increasingly appreciated in the diagnosis, prevention and treatment of eye diseases across the world, Poland included. Recent research has demonstrated the potential of AI systems especially in the field of image recognition,

\section{CORRESPONDING AUTHOR}

Prof. Mirosława Grałek, Department of Pediatric Ophthalmology, M. Konopnicka Pediatrics Center, Independent Public Healthcare Center - Central Teaching Hospital of the Medical University of Lodz, 36/50 Sporna St., 91-738 Lodz, Poland, e-mail: miroslawa.gralek@wp.pl 
which is an intrinsic attribute of ophthalmic evaluation. Artificial neural networks mimic the human brain's pathway for identifying objects by learning pathological features on the basis of supplied collections of digital image datasets. Compared with conventional techniques, ML and DL have been found to be considerably more accurate, even though the current-generation equipment is not yet fully reliable. Deep learning has found applications in the analysis of images obtained by color fundus photography (showing the macula and optic disc) and optical coherence tomography, as well as slit-lamp images, images of tear film layers, automated field of view, and even regular ocular photographs. Methods based on machine learning, and in particular on deep learning, are capable of identifying, locating and evaluating pathological features in almost every disease of the macula and the rest of the retina [3].

In adults, deep learning is most commonly used to detect diabetic retinopathy, macular edema, and age-related macular degeneration, and also in patients with glaucoma [4-7]. In glaucoma, SI is useful mainly for differentiating between the normal optic disc and its pathological (glaucomatous) features, based on available sets of characteristic images, and for detecting the progression of changes in the visual field by analyzing spatial patterns (archetypes) in examined patients over subsequent years of follow-up [8-11]. In 2020, a Polish paper was published, discussing extensive potential applications of AI in preventive screening for glaucoma in adult patients, based on color images of the fundus and intraocular pressure, without any involvement of medical staff [12]. Recently, there have been publications addressing the application of AI in the diagnostic work-up and predicting the course of anterior eye conditions [13]. The assessment of images showing the anterior segment of the eye is standardized according to established algorithms (datasets) analyzed on a 10-point scale (with 10 corresponding to the best, and 0 to the worst scores). The studies have been focusing primarily on the conjunctiva and tear film (dry eye problems), and corneal pathologies.

Pediatric ophthalmology is a branch of ophthalmology which, in recent years, has been increasingly using the potential of AI on account of the specific nature of this ophthalmic field (subspecialty) caused by the young age of the patients and its associated problems $[14,15]$. Ophthalmic evaluation of children, especially the youngest (frequently infants), is challenging due to the lack of cooperation and verbal contact with pediatric patients, while ophthalmic examination under general anesthesia is not always an available option. Other major factors relate to the current shortage of specialists in pediatric ophthalmology and the necessity to procure expensive equipment dedicated to eye examinations in young children. These limitations are found to varying degrees both in countries with advanced healthcare systems and in those with less-developed healthcare infrastructure.

Screening children to determine their visual acuity and establish the causes underlying vision disorders is among the main components of ophthalmic prophylaxis. Early screening tests, performed at the most appropriate time points, allow for quick disease diagnosis and implementation of therapeutic measures that may prevent the impairment or loss of visual acuity and help avoid future vision disability. Between $70 \%$ and $80 \%$ of 19 million children worldwide are affected by vision impairment as a result of a preventable or treatable disease (if detected early enough) [14]. Personal (individual) eye evaluation may be subjective. This is reflected in the interpretation of results which may differ in small details, even if the examination is carried out by experienced ophthalmologists routinely dealing with the management of ocular disorders in children. Such discrepancies may be relevant for making treatment decisions. Diverse interpretations and opinions are especially prevalent in diseases with complex clinical presentations and dynamic progression of changes. Introducing an AI-based system helps avoid the challenge of subjective assessment of visual effects as well as other above-mentioned difficulties involved in the ophthalmic diagnosis of pediatric patients. The aim of the TrackAI project conceptualized and implemented by Pueyo et al. is to develop a system for identifying children with visual disturbances [16]. The program comprises two main elements: an innovative visual test using a neural network installed in a digital device (Device for an Integral Visual Examination, DIVE), adapted to the child's age and physiological visual perception; and SI algorithms operating on a computer device (smartphone) and performing automatic analysis of visual data collected by DIVE. AI algorithms are used to overcome difficulties associated with the interpretation of results of comprehensive vision assessment. It is a multicenter study involving at least five geographically diverse research centers in Europe, the USA, and Asia. The aims of the study are to identify children with reduced visual acuity and to determine the causes of this phenomenon. The study includes children aged from 6 months to 14 years, both with normal and impaired vision development. Overall, at least 2,000 patients will be involved, whose results will be fed to the AI system for "learning" purposes. Over 200 patients from different age groups, both with normal development of vision and various visual pathologies, are enrolled in the validation phase. The results obtained with the aid of AI can be compared with individual patient assessment on the basis of detailed ophthalmic diagnostic evaluation carried out by experienced ophthalmologists. The TrackAI project is currently underway, and high hopes are being pinned on the possibilities it offers, not only by the initiators of the project.

Retinopathy of prematurity (ROP) has invariably been a very important issue in pediatric ophthalmology, related to preterm delivery, and another condition where the benefit of SI in the diagnosis, treatment, and monitoring of childhood eye diseases is clearly recognizable. Worldwide, ROP is the main cause of childhood vision impairment, with a prevalence of $6-18 \%$. In $9 \%$ of preterm babies, it leads to permanent vision loss [4], which makes it an important problem, given a growing number of increasingly immature preterm infants who may develop severe ROP. Early treatment is essential for preserving vision. The clinical features of ROP, taking into account the location of the lesions, their extent and 
advancement as well as the condition of the retinal vessels, are essential for determining whether treatment should be initiated, and selecting the type of therapy. Treatment is indicated after detecting strictly defined abnormalities and the "pre-plus" or "plus" signs manifesting as excessive tortuosity and dilation of the retinal vessels in at least two quadrants of the fundus. Currently, the most common methods for evaluating the retinal vessels and the plus symptom are personalized and subjective in nature. The subjectivity encourages the application of unbiased AI in the diagnosis, screening, and monitoring of therapy in ROP. Another important factor supporting the use of AI is - from the global perspective the lack of specialists trained in problems associated with ROP. Yet another major consideration relates to increasingly frequent claims and complaints for damages filed by parents in situations involving an unfavorable disease course. Such occurrences discourage ophthalmologists from pursuing a professional interest in ROP-related topics. For these reasons, the incorporation of AI into clinical practice for the management of ROP, which was initiated a few years ago, has been gaining momentum recently [17-21]. Current research projects are mainly focused on the assessment of the width of the retinal vessels and their course. These evolving automated assessment methods, which contribute to the objectification of diagnosis through vascular measurements, are becoming the gold standard for the diagnosis of ROP, with a major role in treatment decisions. The datasets on the plus sign are evaluated on the basis of fundus images obtained with the aid of a digital fundus imaging system (RetCam fundus camera). According to Ting et al., the AI-based diagnostic work-up of pre-plus and plus disease - the most important feature of severe ROP - allows objective monitoring of the condition compared to the standard reference diagnostic assessment [8]. The DL algorithms in these studies have achieved 100\% sensitivity and $94 \%$ specificity. Oloui et al. have developed a digital image processing technique to detect retinal blood vessels and measure their tortuosity, and calculate the total length of excessively tortuous vessels in each quadrant and across the entire fundus [17], which has a prognostic significance. Brown et al. have developed software using deep learning technology and a database consisting of 5,511 fundus images taken by a RetCam camera [18]. Studies have shown $93 \%$ sensitivity and $94 \%$ specificity in determining the presence of the plus sign. Recent years have confirmed the benefit of using automated AI based on the principles of deep learning, in the assessment of retinal vascular morphology, and shown that the software being developed can be used as ROP screening tools in premature infants, and improve the objectivity of the diagnostic process [20,21].

In pediatric ophthalmology, $\mathrm{AI}$ also has applications in the diagnosis of cataract and assessment of eligibility for surgical treatment [22-24]. Pediatric cataract is one of the main causes of blindness in the pediatric population; it affects ap- proximately 200,000 children worldwide, with an estimated prevalence of 4.24 per 10,000 live births. At an early stage, cataract is difficult to identify, so AI comes in useful as a method assisting with the detection of the disease in its initial phase, and determining the most suitable time for surgery. The diagnosis of pediatric cataract in an AI-based system, together with the assessment of its structure and stage of maturity, relies on slit-lamp images (photographs) of the lens and uses an artificial convolutional neural network. Lin et al. have developed an AI-based platform called CC-Cruiser designed for the diagnosis of pediatric cataract and providing information on the degree of risk and treatment recommendations [23]. The high accuracy of CC-Cruiser was pre-verified with the use of specific datasets. The project sought to compare the assessments delivered by CC-Cruiser and by ophthalmologists working in real-life clinical settings in terms of diagnostic efficacy and therapeutic decision-making capacity. The study included a total of 350 participants (700 eyes) aged $\leq 14$ years. The findings obtained by the authors show that the accuracy of AI-based diagnosis and decision-making at the current stage is slightly lower than in direct ophthalmic examinations. Despite these limitations, though, CC-Cruiser is a platform that can be useful in the diagnosis of pediatric cataract, reducing the time to diagnosis and eliminating the stress associated with eye examination among the youngest patients. It is the first randomized controlled clinical trial of this type, so before the SI platform is introduced into clinical practice, further work is required to improve the accuracy of the results obtained in the diagnostic work-up of cataract. In addition, the first model for identifying infants at high risk of congenital cataract, i.e. the main cause of preventable childhood blindness, was developed and may be used as an adjunct to the screening procedure [24]. To gain insights into the problems associated with refractive defects of vision, AI algorithms are being developed for myopia, a refractive defect which has been occurring with increasing frequency in young patients. These studies, carried out on the basis of a huge data pool - a total of 687,063 electronic records of patients aged 6-20 years used for elaborating ML algorithms - can predict the development of high myopia, allowing the implementation of preventive measures [25].

The potential applications of AI in selected eye diseases in children presented above, though still limited, are certainly a prelude to further intensive implementation of advanced automated methods of ophthalmic examination. Based on the rapid development of artificial intelligence to date, it is to be anticipated that in the near future AI will bring new computerized diagnostic and therapeutic algorithms with benefits in the treatment of eye diseases in children and adolescents.

\section{DISCLOSURE}

The authors declare no conflict of interest. 


\section{References}

1. Graham N. Artificial Intelligence. Tab Books, Philadelphia 1979.

2. Kaplan A, Michael Haenlein M. Siri, Siri, in my hand: Who's the fairest in the land? On the interpretations, illustrations, and implications of artificial intelligence. Bus Horiz 2019, 62: 15-25.

3. Schmidt-Erfurth U, Sadeghipour A, Gerendas B, et al. Artificial intelligence in retina. Prog Retin Eye Res 2018; 67:1-29.

4. Keskinbora K, Güven F. Artificial Intelligence and Ophthalmology. Turk J Ophthalmol 2020; 50:37-43.

5. Gulshan V, Peng L, Coram M, et al. Development and validation of a deep learning algorithm for detection of diabetic retinopathy in retinal fundus photographs. JAMA 2016; 316: 2402-2410.

6. Padhy SK, Takkar B, Chawla R, et al. Artificial intelligence in diabetic retinopathy: A natural step to the future. Indian J Ophthalmol. 2019; 67: 1004-1009.

7. Hwang DK, Hsu Ch-Ch, Chang K-J, et al. Artificial intelligence-based decision-making for age-related macular degeneration. Theranostics. 2019; 9: 232-245.

8. Ting DSW, Pasquale LR, Peng L, et al. Artificial intelligence and deep learning in ophthalmology. Br J Ophthalmol 2019; 103: 167-175.

9. Bajwa MN, Malik MI, Siddiqui SA, et al. Two-stage framework for optic disc localization and glaucoma classification in retinal fundus images using deep learning. BMC Med Inform Decis Mak 2019; 19: 136.

10. Wang $M$, Shen $L Q$, Pasquale $L R$, et al. An artificial intelligence approach to detect visual field progression in glaucoma based on spatial pattern analysis. Invest Ophthalmol Vis Sci. 2019; 60: 365-375.

11. Barros DMS, Moura JCC, Freire CR, et al. Machine learning applied to retinal image processing for glaucoma detection: review and perspective. Biomed Eng Online 2020; 19: 20.

12. Zaleska-Żmijewska A, Szaflik JP, Borowiecki P, et al. A new platform designed for glaucoma screening: identifying the risk of glaucomatous optic neuropathy using fundus photography with deep learning architecture together with intraocular pressure measurements. Klin 0czna 2020; 122: 1-6.

13. Wu X, Liu L, Zhao L, et al. Application of artificial intelligence in anterior segment ophthalmic diseases: diversity and standardization. Ann Transl Med 2020; 8: 714

14. Natarajan S. Pediatric ophthalmology: The oldest ophthalmology subspeciality. Indian J Ophthalmol 2011;59: 419-420.

15. Reid JE, Eaton E. Artificial intelligence for pediatric ophthalmology. Curr Opin Ophthalmol 2019; 30:337-346.

16. Pueyo V, Pérez-Roche T, Prieto E, et al. Development of a system based on artificial intelligence to identify visual problems in children: study protocol of the TrackAl project. BMJ Open 2020; 10: e033139.

17. Oloumi F, Rangayyan RM, Ells AL. Computer-aided diagnosis of retinopathy in retinal fundus images of preterm infants via quantification of vascular tortuosity. J Med Imaging (Bellingham) 2016; 3:044505.

18. Brown JM, Campbell JP, Beers A, et al. Automated diagnosis of plus disease in retinopathy of prematurity using deep convolutional neural networks. JAMA Ophthalmol 2018; 136: 803-810.

19. Redd TK, Campbell JP, Brown JM, et al. Evaluation of a deep learning image assessment system for detecting severe retinopathy of prematurity. Br J Ophthalmol 2018; bjophthalmol-2018-313156.

20. Tan Z, Simkin S, Lai C, et al. Deep learning algorithm for automated diagnosis of retinopathy of prematurity plus disease. TransI Vis Sci Technol 2019; 8: 23.

21. Scruggs BA, Chan RVP, Kalpathy-Cramer J, et al. Artificial intelligence in retinopathy of prematurity diagnosis. TransI Vis Sci Technol 2020; 9:5.

22. Liu X, Jiang J, Zhang K, et al. Localization and diagnosis framework for pediatric cataracts based on slit-lamp images using deep features of a convolutional neural network. PLoS One 2017; 12: e0168606.

23. Lin H, Li R, Liu Z, et al. Diagnostic efficacy and therapeutic decision-making capacity of an artificial intelligence platform for childhood cataracts in eye clinics: A Multicentre randomized controlled trial. EClinicalMedicine 2019; 9: 52-59.

24. Lin D, Chen J, Lin Z, et al. A practical model for the identification of congenital cataracts using machine learning. EBioMedicine 2020; 51: 102621.

25. Lin $\mathrm{H}$, Long $\mathrm{E}$, Ding $\mathrm{X}$, et al. Prediction of myopia development among Chinese school-aged children using refraction data from electronic medical records: A retrospective, multicentre machine learning study. PLoS Med 2018; 15: e1002674. 